\title{
X-Ray Photoacoustic Imaging of Surface and Subsurface Layers of Structured Materials
}

\author{
Kenji KaTo* and Yoshinori SuglTaNI** \\ *National Chemical Laboratory for Industry, Higashi, Tsukuba 305, Japan \\ **Department of Materials Science, Kanagawa University, Tsuchiya, Kanagawa 259-12, Japain
}

\begin{abstract}
Surface and subsurface layers of structured materials were observed as the amplitude and phase images of X-ray photoacoustic signals. An X-ray beam from synchrotron radiation was narrowed with the use of a lead slit and allowed to irradiate the sample in the photoacoustic cell. A laminated sheet of aluminum and lead foils and a flexible print circuit board were used as samples. For the laminated sample, the empty space in the subsurface lead layer was clearly visualized in the images of both amplitude and phase delay. The results were compared with those of the photoacoustic measurement in the visible region. For the flexible print-circuit board, the printed copper strips were observed in both the amplitude and the phase images. The circuit pattern below the surface polymer layer was observed as signals with large phase delays.
\end{abstract}

Keywords X-Ray, photoacoustic measurement, imaging, subsurface structure, layered material

Photoacoustic signals depend on the optical, thermal and elastic properties as well as on the geometrical shape of the sample. ${ }^{1}$ Photoacoustic imaging techniques for characterizing structured samples have been widely investigated. ${ }^{2}$ Particularly, non-destructive observation of the surface and subsurface structure is of interest in characterizing materials such as ceramic coatings $^{3}$, semiconductors ${ }^{4,5}$, subsurface grooves ${ }^{6}$ and subsurface defects of ceramics. ${ }^{7}$ In recent years, it has been important to extend the wavelength region of the photoacoustic measurement into the $\mathrm{X}$-ray region. ${ }^{8} \mathrm{X}$ Ray photoacoustic measurements are similar to those in the visible region in the sense that the thermal properties of samples are reflected on the formation of the image. Since X-rays penetrate deeply into materials, the measurement of subsurface structures seems to be more informative in the $\mathrm{X}$-ray region than in the visible region. Furthermore, samples show different optical properties in the $\mathrm{X}$-ray region than in the visible region, consequently, it is of interest to measure the photoacoustic images in both of the wavelength regions for comparing the two images.

\section{Experimental}

Experiments in the X-ray region were conducted using synchrotron radiation at beam line BL-15A of the Photon Factory (Tsukuba, Ibaraki, Japan). The beam was monochromated at $1.504 \AA$. Figure 1 shows a schematic diagram of the experimental setup. The $\mathrm{X}$-ray beam was narrowed with the use of a lead slit
$1 \mathrm{~mm}$ in diameter and was chopped with a rotating sector. The photoacoustic cell used in this experiment was the same as that used in the previous works., ${ }^{9,10}$ The cell windows and the cell body were made of beryllium and brass, respectively. The photoacoustic cell was mounted on an $X Z$-stage controlled by a desktop computer (NEC, PC9801). The photoacoustic signal was detected by a condenser microphone and then amplified by a lock-in amplifier (NF CIRCUIT

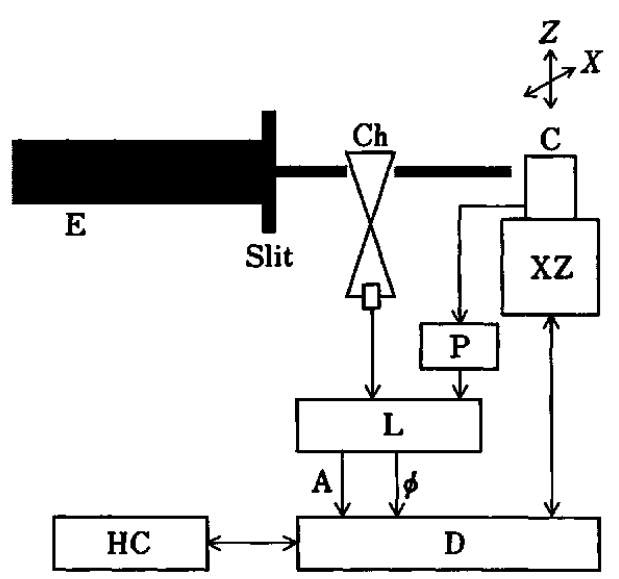

Fig. I Schematic diagram of the experimental setup for an X-ray photoacoustic imaging. $E$, monochromated X-ray beam of synchrotron radiation; $\mathrm{Ch}$, chopper; $\mathrm{C}$, photoacoustic cell; $\mathrm{XZ}$, computer controlled $X Z$-stage; $\mathrm{P}$, preamplifier; L, lock-in amplifier; $D$, desk top computer with $A / D$ converter; HC, hardcopy. 
BLOCK INC., LI575). The amplitude and phase output signals of the lock-in amplifier were led to an analog-to-digital converter and stored in the computer memory. Data acquisition time for each data point was required to be 30 to $60 \mathrm{~s}$ to obtain a desirable photoacoustic image, because the X-ray beam was narrowed by the lead slit and thus its intensity was weak. Each step of the $X Z$-stage was set to $1 \mathrm{~mm}$ or $0.5 \mathrm{~mm}$. In the former case, the imaging area was divided into $16 \times 16$ points (256 pixels) and in the latter case the imaging area was divided into $32 \times 32$ points (1024 pixels). In the case of photoacoustic measurements with visible light excitation, the upper beryllium window of the cell was replaced with a quartz window $1 \mathrm{~mm}$ thick. The light beam from a $500 \mathrm{~W}$ xenon arc lamp was filtered with an UV cut (TOSHIBA, Y44) and an IR cut (TOSHIBA, IRA-25S) filters and focused to a spot of about $1 \mathrm{~mm}$ in diameter on the specimen.

\section{Samples}

A laminated sheet of aluminum and lead foil and a flexible print circuit board were used as samples. In the case of the laminated sample, pieces of aluminum foil (thickness about $15 \mu \mathrm{m}$, diameter $18 \mathrm{~mm}$ ) were made to adhere on the two sides of a piece of lead foil (thickness $100 \mu \mathrm{m}$, diameter $18 \mathrm{~mm}$ ) by cyanoacrilate resin. The lead layer had a rectangular hole $(3 \times 4 \mathrm{~mm})$ at the center. The flexible print circuit board consisted of basically 3 layers: i) orange colored polymer base, ii) copper print circuit strip or colorless polymer layers on both sides of the base, and iii) orange colored surface polymer layers on both sides of the print circuit board. The thickness of each layer was about $50 \mu \mathrm{m}$. The bare part of the print pattern of copper strip was coated with soft metallic solder.

\section{Results and Discussion}

Figures 2(a) and 2(b) show the X-ray photoacoustic images of amplitude and phase, respectively, of the laminated sample made of aluminum and lead foils, while Figs. 2(c) and 2(d) show the similar photoacoustic images of the same sample measured by visible light excitation. The chopping frequency was $10 \mathrm{~Hz}$. The empty parts of the lead foil below the surface layer are clearly seen in the figure. In the amplitude image (Fig. 2(a)), signal intensity is weak in the central part of the specimen and strong in its surroundings. On the contrary, signal intensity for the visible light excitation is strong at the central part and weak in its surroundings (Fig. 2(c)). These results are interpreted as follows: i) X-ray absorption mainly occurs in the lead foil, and consequently the signal intensity is weak in the central part where the lead foil is missing; ii) The absorption of visible light occurs only in the surface of the aluminum foil, so that the heat will be generated uniformly over the whole surface of the specimen. The thermal property, however, is not uniform over the whole
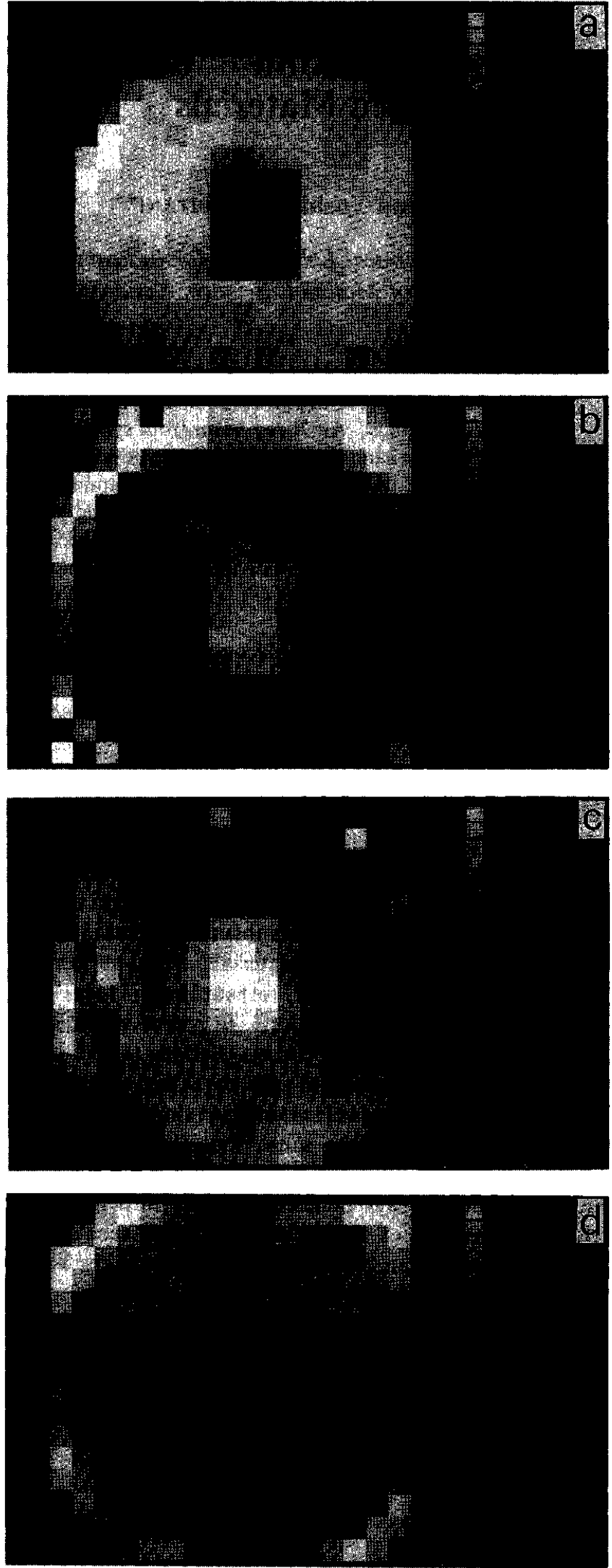

Fig. $2 \mathrm{X}$-ray photoacoustic and visible light photoacoustic images of the laminated sample of lead foil and aluminum foils. a, X-ray amplitude image; b, X-ray phase image; c, visible light amplitude image; d, visible light phase image. 
surface due to the presence of the lead foil beneath, and the heat loss due to the heat diffusion into the lead foil occurs only in the peripheral part of the sample. Consequently, the photoacoustic signal is intense in the central part of the sample.

In the X-ray photoacoustic image of the phase signal (Fig. 2(b)), the phase delay is small in the central part of the specimen and large in its surroundings. On the contrary, in visible light excitation, phase delay is large in the central part of the specimen and small in its
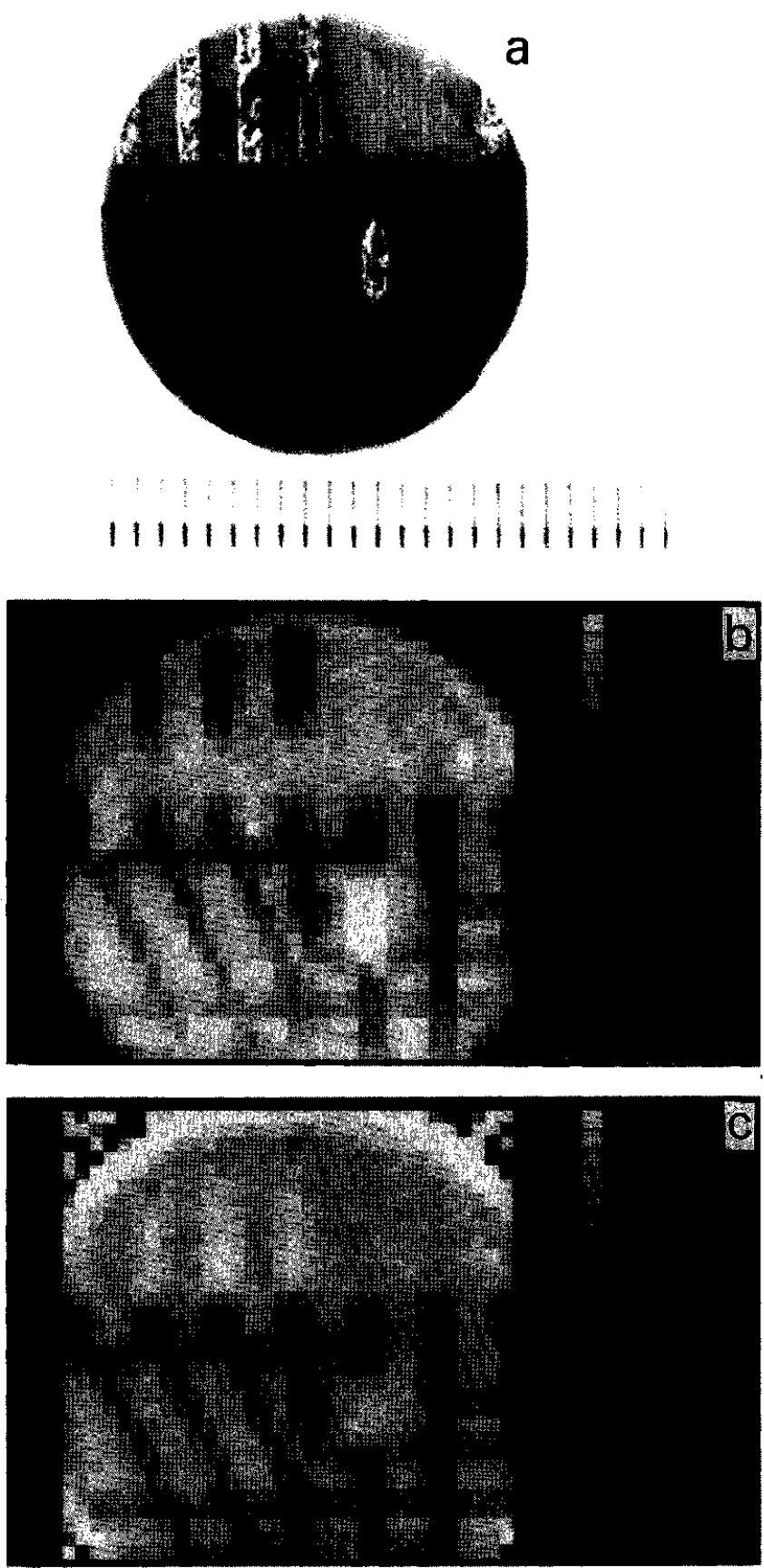

Fig. 3 X-ray photoacoustic images and photograph of the flexible print circuit board. a, photograph of the side faced to $X$-ray beam ( 1 div. $=1 \mathrm{~mm}$ ); $b$, amplitude image; $c$, phase image. surroundings (Fig. 2(d)). These results are also interpreted as follows: i) In the central part of the specimen, the X-ray absorption occurs only in the surface aluminum layer and the phase delay is small. In the surrounding part of the specimen, X-ray absorption mainly occurs in the lead foil which is at a deeper level of the specimen and consequently the phase delay is large. ii) Absorption of visible light occurs over the whole surface of the aluminum foil. In its surroundings, however, the heat loss occurs due to heat diffusion in the lead foil, resulting in the immediate decrease of the surface temperature, so that the heat delay is larger in the central part and smaller in the peripheral, cool part of the specimen.

The results obtained in the experiments above show that the mechanism of the photoacoustic signal with $\mathrm{X}$ ray excitation is somewhat different from that with visible light excitation: in the imaging experiment with $\mathrm{X}$-ray excitation, the obtained images reflect the variation of the amount of heat generated in the specimen, while those with visible light excitation reflect the variation of the rate of heat diffusion in the specimen.

Figure 3 shows a photograph (a) and X-ray photoacoustic images $(b, c)$ of the flexible print-circuit board. The chopping frequency was $29 \mathrm{~Hz}$. The step of the $X Z$-stage was $0.5 \mathrm{~mm}$. The printed copper stripes coated with solder were observed in the images of signal amplitude and phase delay. The print-circuits in the lower part of the specimen is covered with polymer coating, which are also seen in the intensity and the phase images. In the image of phase delay, printcircuits on the surface showed small phase delay. On the other hand, the print circuits at the subsurface layer were observed to have patterns corresponding to a large phase delay.

The authors would like to thank Dr. Nobuyuki Amemiya of the National Laboratory for High Energy Physics for his help concerning the X-ray measurements at the beam line of the photon factory. The present work was partially supported by Grant-in-Aid for Scientific Research No. 61840018 from the Ministry of Education, Science and Culture.

\section{References}

1. A. Rosencwaig, "Photoacoustics and Photoacoustic Spectroscopy", John Wiley \& Sons, New York, 1980.

2. A. Rosencwaig, J. Appl. Phys., 51, 2210 (1980).

3. M. Liezers and R. M. Miller, "Springer Series in Optical Sciences Vol. 58, Photoacoustic and Photothermal Phenomena", p. 437, Springer Verlag, Berlin, 1987.

4. N. Mikoshiba, H. Nakamura and K. Tsubouchi, Oyo Butsuri, 58, 483 (1985).

5. K. Kubota and H. Murai, J. Appl. Phys., 56, 835 (1984).

6. M. Izuchi, H. Nakano and S. Nagai, Jpn. J. Appl. Phys., 26, 221 (1987).

7. I. Tomeno and H. Ohzu, Jpn. J. Appl. Phys., 24, 1445 (1985).

8. K. Kato and Y. Sugitani, Anal. Sci., 3, 275 (1987). 
9. K. Kato and Y. Sugitani, Anal. Sci., 5, 545 (1989).

10. K. Kato and Y. Sugitani, Anal. Sci., 6, 177 (1990).

(Received October 24, 1990) (Accepted December 22, 1990) 